\title{
New Directions for Low Dimensional Thermoelectricity
}

M. S. Dresselhaus ${ }^{(a, b)}$ Y. M. Lin, ${ }^{(b)}$ M. R. Black, ${ }^{(b)}$ O. Rabin, ${ }^{(c)}$ G. Dresselhaus ${ }^{(d)}$

Massachusetts Institute of Technology, Cambridge, MA 02139

(a) Department of Physics,

(b) Department of Electrical Engineering and Computer Science,

(c) Department of Chemistry,

(d) Francis Bitter Magnet Laboratory

\begin{abstract}
Low dimensionality provides opportunities to modify the properties of bulk materials dramatically and to control materials properties independently in a manner that is not possible for bulk materials. The special characteristics of low dimensional materials to enhance thermoelectric performance have already been demonstrated in quantum wells, quantum wires and quantum dots. The main focus of this review is a summary of advances made in the modeling of quantum dot superlattice nanowires. Several new research directions for low dimensional thermoelectricity or inspired by this research are briefly mentioned.
\end{abstract}

\section{INTRODUCTION}

Low-dimensional systems, such as 1D quantum wires and 2D superlattices, have shown new opportunities for these systems to control and enhance thermoelectric parameters independently, in a manner not possible for bulk materials. Due to their large surface to volume ratio, enhanced surface and interface effects and quantum confinement effects, nanostructured materials are expected to exhibit and in some cases have actually shown, dramatically different thermal and electronic transport behaviors that are absent in their bulk counterparts, with distinct opportunities for enhanced thermoelectric performance. In this context, Hicks and Dresselhaus [1,2] predicted enhanced thermoelectric performance in $1 \mathrm{D}$ and 2D systems compared to their counterpart bulk materials, due to both a higher density of states in low-dimensional systems for enhanced thermopower and an increased phonon scattering or reduced lattice thermal conductivity, as recently demonstrated by Venkatasubramanian [3] through enhanced interface scattering in short period superlattices. Thermoelectric cooling based on nanostructures has other advantages, in addition to improved static thermoelectric performance, in that a very small active region with a much shorter response time than those of bulk thermoelectric devices or conventional mechanical cooling systems can be achieved, which is of great interest for modern microelectronics where the efficiency bottleneck is usually limited by local hot spots. It is expected that by integrating nanostructured thermoelectric materials into critical regions of microelectronic circuits, the excess heat that limits the device performance can be effectively removed.

Theoretical calculations and experimental investigation of the thermoelectric properties of $2 \mathrm{D}$ and $1 \mathrm{D}$ systems have been studied extensively for various materials [3-5]. Since the enhancement in thermoelectric properties is anticipated to be more pronounced as the dimensionality decreases, 0D or quantum dot structures are promising candidates for seeking 
further improvement beyond that shown in 1D systems due to increased quantum confinement and interface scattering. Model 0D structures for thermoelectricity are the focus of this paper. However, unlike 1D or 2D systems, where at least one of the directions is not quantum-confined and thus can provide electrical conduction, 0D structures are confined in all directions and this may present difficulties for maintaining efficient electrical transport channels by tunneling or hopping transport between neighboring dots. For this purpose, several novel structures based on quantum dots that capture the essence of 0D structures, while still enabling transport phenomena, have been proposed and synthesized, such as quantum dot array superlattices [6] and superlattice nanowires [7]. Quantum dot structures have already shown great promise as having demonstrated an impressive thermoelectric performance of $Z T \sim 2$ at $300 \mathrm{~K}$ for PbTeSe-based quantum dot superlattices [6], compared to a highest $Z T$ of $\sim 1$ for conventional bulk materials. A possible mechanism for this enhanced thermoelectric performance has been proposed [8] to be due to mini-band formation in coupled $3 \mathrm{D}$ quantum dot arrays.

In the present review, a summary of recent advances in the modeling of quantum dot superlattice nanowires is presented along with comments about recent advances and new research directions for this field.

\section{Superlattice Nanowires}

Quantum dot nanostructures of interest as model structures for 0D thermoelectricity are superlattice nanowires (SLNWs), shown in Fig. 1(a), which consist of a series of interlaced nanodots of two different materials, as denoted by $A$ and $B$. In the superlattice nanowire (SLNW) structure, the electronic transport along the wire axis is made possible by the tunneling between adjacent quantum dots, while the uniqueness of each quantum dot and its $0 \mathrm{D}$ characteristics are maintained by the energy difference of the conduction or valence bands between different materials [see Fig. 1(b)]. The band offset $\Delta E_{n m}$ not only provides some amount of quantum confinement, but also creates a periodic potential for carriers moving along the wire axis, which may result in both a larger and sharper density of electronic states than is present in ordinary 1D systems, as well as a reduced the lattice thermal conductivity by blocking the phonon conduction along the wire axis [9]. In the superlattice nanowire, electrical conduction may be sustained and may benefit from the unusual electronic band structures due to the periodic potential perturbation [10].

Various approaches have been developed to synthesize superlattice nanowire structures with different materials. $\mathrm{Co} / \mathrm{Cu}$ superlattice nanowires were first fabricated in nanoporous templates using electrochemical deposition [7], and more recently, researchers have synthesized highly-crystalline semiconductor superlattice nanowires (e.g., Si/SiGe [11], GaAs/GaP [12], and InP/InAs [13]) by the Vapor-Liquid-Solid growth mechanism. Due to the structural complexity and the material diversity in these quantum-dot-based systems, it is essential to develop a model to understand the behavior and to predict properties of interest in these novel structures, especially for practical applications and device optimization. In the following, we briefly summarize results on a theoretical model for the electronic structure and transport properties of superlattice nanowires, which explicitly takes into account the cylindrical wire boundary conditions, the diameter-dependent band offsets for the periodic potential along the wire axis, and the multiple anisotropic carriers, and consideration is given to variation of materials parameters for thermoelectric applications. 
(a)

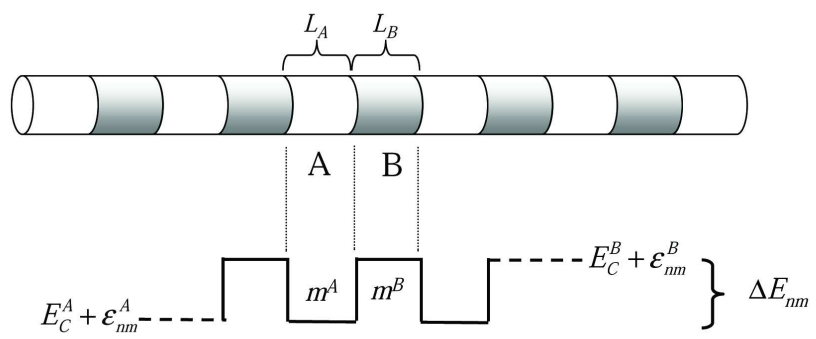

(b)

Figure 1: (a) Schematic diagram of a superlattice nanowire of diameter $d_{w}$ consisting of interlaced nanodots $A$ and $B$. (b) Schematic potential profile of one conduction subband in a superlattice nanowire with a band offset $\Delta E_{n m}[10]$.

The transport and electronic properties of superlattice nanowires are determined by their electronic band structure, which exhibits dramatically different features from that of a simple 1D nanowire or of bulk materials. To model the electronic band structure of superlattice nanowires, we assume that each segment has a circular cross section with a uniform wire diameter $d_{w}$ [see Fig. 1(a)], and the carriers in each cylindrical nanodot are confined by an infinite potential at the wire boundary (the cylindrical sidewall), which is a good approximation for nanowires embedded in a wide bandgap insulating matrix or for free-standing nanowires. We also assume that each nanodot consists of a sufficient number of atoms so that the effective mass theorem is valid to describe the local electronic properties of a nanodot by the band structure parameters of the bulk material.

The $z$ axis is chosen to be along the wire axis, and we first consider the electronic structure in one nanodot (material $A$ for example). Due to the quantum confinement normal to the wire axis, quantized subbands are formed in the nanodot. These electrons are described by a wave function, which exhibits unrestricted motion for electrons in the $z$ direction within the nanodot and has quantum confinement in the $x, y$ plane. The corresponding energy eigenvalues include terms $E_{C}^{A}$ for the energy of the conduction band edge for bulk material $A$, a quantization energy $\epsilon_{n m}^{A}$ of the $(n, m)$ subband, and plane wave energies $\hbar^{2} k^{2} / 2 m_{z}^{A}$ where $m_{z}^{A}$ is the transport effective mass along the wire axis. The quantization energy $\epsilon_{n m}$ and corresponding wave function both depend on the geometry of the wire cross section, the wire diameter, and the carrier effective masses, and anisotropic carrier masses are explicitly considered.

By arranging the two types of nanodots $(A$ and $B)$ into a superlattice nanowire [see Fig. 1(a)], the electrons in the $(n, m)$ subband will experience a periodic square-well potential $U(z)$ with an energy barrier height $\Delta E_{n m}$ [see Fig. 1(b)] that depends on both the bandgap of the bulk material and the quantization subband energies $\epsilon_{n m}$ for both materials A and B [10]. Although independent quantum dots possess discrete energy levels like that of atoms, superlattice nanowires exhibit 1D-like dispersion relations along the wire axis due to the wave function leakage across the finite barrier height $\Delta E_{n m}$ between quantum dots. The (sub)band offset energies $\Delta E_{n m}$ are usually different from that of bulk materials $\left(E_{C}^{A}-E_{C}^{B}\right)$ due to the quantum confinement energy $\epsilon_{n m}$ for each type of quantum dot, and $\Delta E_{n m}$ may also depend on the subband index $(n, m)$ and the wire diameter $d_{w}$.

Making use of the Bloch Theorem associated with the periodic potential $U(z)$ along the 
wire axis and by matching of the wave functions and their derivatives across the boundaries of the superlattice nanowire, the energy eigenvalue problem is solved [10]. Let us first examine the electronic density of states of a subband for the superlattice nanowire, $D(E)$ and its integral over $E$ giving the number of states up to energy $E$. The results are shown in Fig. 2 for nanowires with various segment lengths $\left(L_{A}=L_{B}=1,5,20\right.$, and $\left.60 \mathrm{~nm}\right)$, assuming a potential barrier height of $V_{b}=50 \mathrm{meV}$ and an effective mass of $m_{z}^{A}=m_{z}^{B}=0.5 m_{0}$, where $m_{0}$ is the free electron mass. The zero in energy is chosen to be at the top of the potential barrier (see Fig. 1). It is found in Fig. 2(a), that for very short superlattice periods $\left(L_{A}=1 \mathrm{~nm}\right)$, the electronic band structure approaches that of an alloy system (Alloy limit) with a density of states similar to that of a simple 1D system. In this limit, the onset of the density of states corresponds to an effective band edge in the middle of the potential well, and this effective band edge is equal to the average of the periodic potential [see Fig. 2(a)]. As the segment length increases, the density of states (or the number of states) begins to develop mini-bands and mini-gaps in the subband structure [see Figs.2(b) and (c)], exhibiting features unique to the superlattice nanowires and not seen in conventional 1D nanowires. The widths of these mini-bands are usually very narrow $[\sim 1 \mathrm{meV}$ for the first mini-band in Fig. 2(b)] for energies below the potential well $(E<0)$, yielding a $\delta$-functionlike density of states in the potential well, as represented by vertical lines in Figs.2(b) and (c). These sharp features in $D(E)$ are similar to the discrete states of quantum dots. It is found that the width of these mini-bands generally increases and the mini-band gap decreases with increasing energy. We also note that the number of these narrow mini-bands in the potential well increases with increasing segment length [see Fig. 2(c)]. While the mini-bands here are well-separated in energy for states with energies in the potential well $(E \leq 0)$, the electronic band structure approximates the continuum of the classical limit for electrons with energies above the potential barrier $(E>0)$. Finally, for long segment lengths $\left(L_{A} \geq 60 \mathrm{~nm}\right)$, these mini-bands become so close to each other in energy that they may be approximated by a continuum. If the mini-gaps are much smaller than the thermal energy [see Fig. 2(d)], the density of states of the superlattice nanowire is expected to be approximately equivalent to the averaged density of states of the two constituent materials (Classical limit).

The dramatically different electronic $D(E)$ of superlattice nanowires shown in Figs. 2(a)(d) can be qualitatively categorized, based on a comparison of several length and energy scales, namely, the electron de Broglie wave length $\Lambda_{e}$ which is approximately $2 \pi \hbar / \sqrt{2 m_{z}^{A} V_{b}}$, the segment length $\left(L_{A}\right.$ or $L_{B}$ where $\left.L=L_{A}+L_{B}\right)$, the mini-gap energy $\left(\epsilon_{g}\right)$, which can be approximated by the energy separation between two mini-bands which is approximately $(\pi \hbar / L) \sqrt{V_{b} / 2 m_{z}^{A}}$ in which $V_{b}$ is the potential barrier height. When the segment length is much shorter than the de Broglie wavelength, a full electronic wavelength would cover many superlattice periods $L$, so that the electrons only experience an averaged potential without noticing the detailed potential structure as they travel along the nanowire (Alloy limit). On the other hand, if the thermal energy $k_{B} T$ is much larger than the mini-gap energy $\epsilon_{g}$, the mini-bands can be treated as continuous states due to the thermal smearing (Classical limit). The density of states in these various limits is used to calculate the Seebeck coefficient and the electrical conductivity [10] using similar approximations as has been used previously for low dimensional systems [5].

The phonon transport in superlattice nanowires is also strongly influenced by additional 


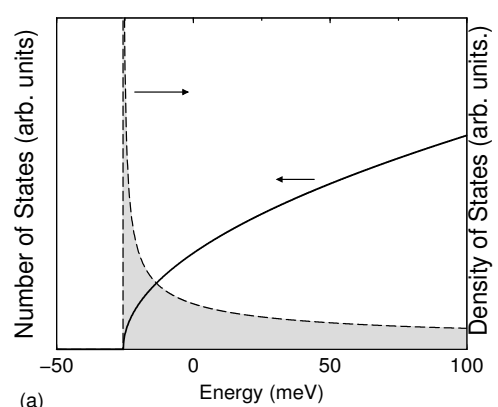

(a)

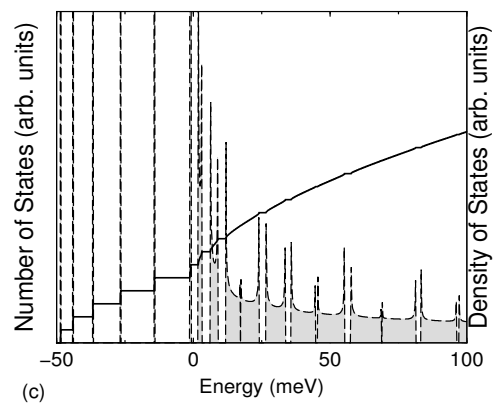

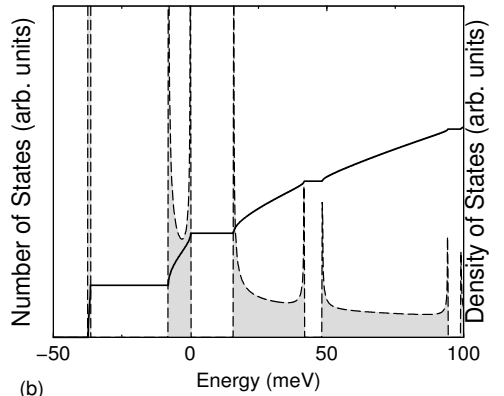

(b)

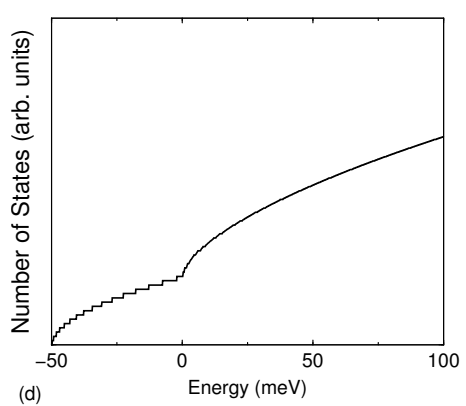

Figure 2: Calculated density of states and the number of states of a 1D subband in a periodic potential with different segment lengths $L_{A}=L_{B}=$ (a) $1 \mathrm{~nm}$, (b) $5 \mathrm{~nm}$, (c) $20 \mathrm{~nm}$, and (d) $60 \mathrm{~nm}$, assuming a potential barrier height of $50 \mathrm{meV}$ and effective masses of 0.5 $m_{0}$. The zero in energy is chosen to be at the top of the potential barrier [10].

phonon scattering at the wire boundary and at the interfaces between the quantum dots. The lattice thermal conductivity should be significantly reduced as the wire diameter or the segment length becomes smaller than the bulk phonon mean free path. The lattice thermal conductivity for superlattice nanowires has been modeled, based on the phonon Boltzmann transport equations with diffuse mismatch interface conditions [9]. The results of these calculations are used for modeling the lattice contribution to the thermal conductivity while the electronic contribution is obtained from use of the Wiedemann-Franz relation [10]. The general derivations for superlattice nanowires are appropriate for a variety of materials properties and for materials in general [10].

We now show the effect of variation of the superlattice parameters on the thermoelectric figure of merit of superlattice nanowires by the consideration of a specific materials system, namely the lead chalcogenide family ( $\mathrm{PbTe}, \mathrm{PbSe}$, and $\mathrm{PbS}$ ). This materials family is an attractive model system to study the effect of the superlattice structure on the thermoelectric properties of nanowires, because of their simple crystal and electronic structures with materials properties that are well known over a wide temperature range. The constant energy surfaces for the conduction and valence bands of these lead salts are prolate ellipsoids of revolution centered at the four equivalent $L$ points in the Brillouin zone and the major axes of these ellipsoids are in the [111] directions.[14] The lead salts are chosen for our model studies of superlattice nanowires because they are good thermoelectric materials in bulk form with well-established transport properties, and have been demonstrated to make effective quantum dot thermoelectric materials [6]. The thermoelectric properties of these lead salt superlattice nanowires, particularly $\mathrm{PbS} / \mathrm{PbSe}$, are investigated to elucidate their performance dependence on the segment length, wire diameter, crystal orientation along the 


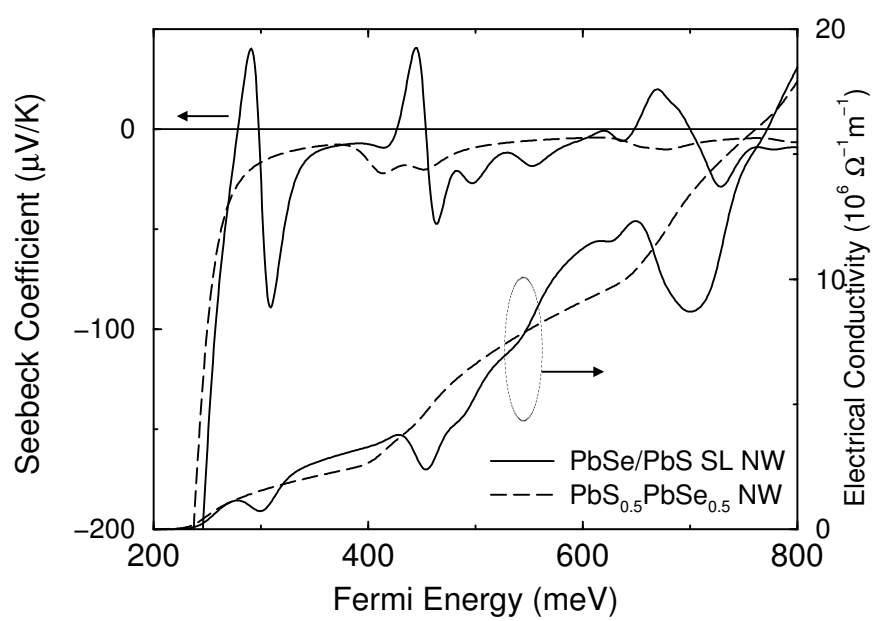

Figure 3: Calculated $S$ and $\sigma$ as a function of $E_{F}$ for [001] $\mathrm{PbSe}(5 \mathrm{~nm}) / \mathrm{PbS}(5 \mathrm{~nm})$ superlattice and $\mathrm{PbS}_{0.5} \mathrm{Se}_{0.5}$ alloy nanowires at $77 \mathrm{~K}$. The wire diameters are both $d_{w}=10 \mathrm{~nm}$, and the zero of energy is as in Fig. 2 [10].

wire axis, and the length ratio of the constituent nanodots. The thermoelectric performance is usually measured in terms of a dimensionless figure of merit $Z T$ defined as

$$
Z T=\frac{S^{2} \sigma}{\kappa} T
$$

where $S, \sigma$, and $\kappa$ are the Seebeck coefficient, electrical conductivity, and the thermal conductivity (including contributions from electrons and phonons), respectively.

Figure 3 shows the calculated Seebeck coefficient and electrical conductivity as a function of Fermi energy for [001] $\mathrm{PbSe} / \mathrm{PbS}$ superlattice nanowires with a diameter $d_{w}=10 \mathrm{~nm}$ and segment lengths $L_{\mathrm{PbS}}=L_{\mathrm{PbSe}}=5 \mathrm{~nm}$ at $77 \mathrm{~K}$, as well as the calculated results for $\mathrm{PbS}_{0.5} \mathrm{Se}_{0.5}$ alloy nanowires with the same diameter and crystal orientation. The relaxation times $\tau$ of electrons and holes, required for calculating various transport-related integrals are derived from the carrier mobility of bulk materials [15] using the relation $\mu=e \tau / \mathrm{m}^{*}$, as listed in Table 1 for various lead salts at $77 \mathrm{~K}$. However, the carrier mobility of lowdimensional systems may possess different values from those of their bulk counterparts. It is expected that $\mu$ may be lowered due to the extra scattering at the wire boundary and the heterogeneous interfaces, whereas the reduction in available final states for scattering events in low-dimensional systems may increase $\mu$. Therefore, careful experimental studies are required to obtain a more accurate estimate for $\mu$ and its temperature dependence and to improve the predictive capabilities of model calculations.

As shown in Fig. 3, the electrical conductivity of a homogeneous (alloy) nanowire increases monotonically with increasing Fermi energy (or electron density), while that for the superlattice nanowires exhibits non-monotonic variations with local minima corresponding to the mini-gaps in the density of states (see Fig.2). In Fig.3, the Seebeck coefficient of superlattice nanowires also shows unusual behaviors due to the existence of the mini-gaps. We note that for alloy nanowires, the Seebeck coefficient is always negative and the magnitude diminishes rapidly with increasing Fermi energy, except for some small fluctuations near the subband edges. Interestingly, for superlattice nanowires, the Seebeck coefficient not only shows strong oscillations near the mini-gaps, but $S$ also becomes positive for certain 
Table 1: Mobility of electrons and holes for various lead salts at $77 \mathrm{~K}$.[15] The carrier relaxation time is derived using the relation $\tau=m^{*} \mu / e$.

\begin{tabular}{l|c|c|c}
\hline \hline Property, unit & PbS & PbSe & PbTe \\
\hline$\mu_{e}(77 \mathrm{~K}), \mathrm{cm}^{2} \mathrm{~V}^{-1} \mathrm{~s}^{-1}$ & 11000 & 16500 & 31600 \\
$\mu_{h}(77 \mathrm{~K}), \mathrm{cm}^{2} \mathrm{~V}^{-1} \mathrm{~s}^{-1}$ & 15000 & 13700 & 21600 \\
$\tau_{e}(77 \mathrm{~K}), \mathrm{s}$ & $5.83 \times 10^{-13}$ & $5.11 \times 10^{-13}$ & $8.86 \times 10^{-13}$ \\
$\tau_{h}(77 \mathrm{~K}), \mathrm{s}$ & $7.62 \times 10^{-13}$ & $3.73 \times 10^{-13}$ & $7.13 \times 10^{-13}$ \\
\hline \hline
\end{tabular}

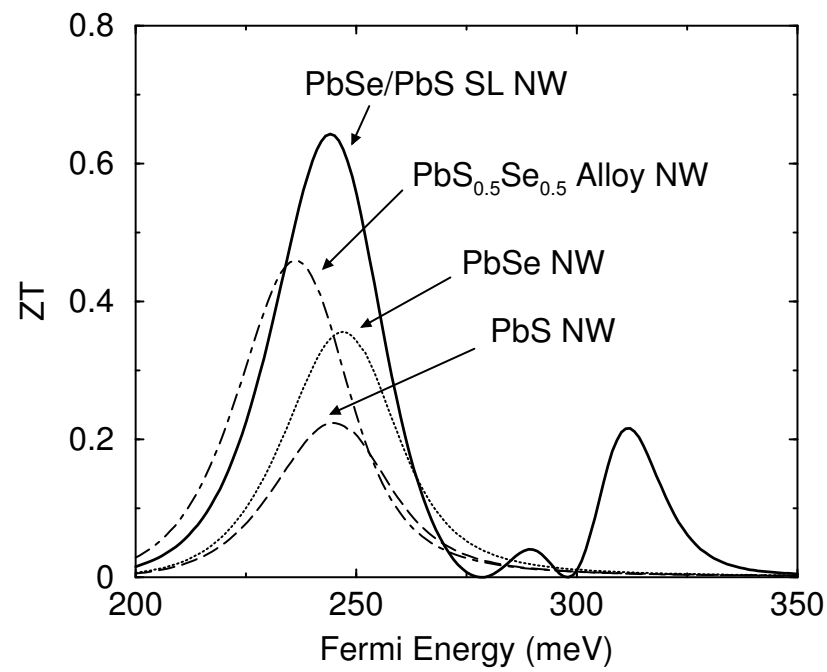

Figure 4: Calculated dimensionless figure of merit as a function of Fermi energy for an [001] $\mathrm{PbSe}(5 \mathrm{~nm}) / \mathrm{PbS}(5 \mathrm{~nm})$ superlattice, and $\mathrm{PbSe}, \mathrm{PbS}$, and $\mathrm{PbS}_{0.5} \mathrm{Se}_{0.5}$ alloy nanowires at $77 \mathrm{~K}$. The wire diameters are all $d_{w}=10 \mathrm{~nm}[10]$.

energy ranges. This sign change in the Seebeck coefficient as the Fermi energy varies has important implications, indicating that superlattice nanowires may be tailored to exhibit $n$ or $p$-type properties, using the same dopants (e.g., electron donors) by carefully controlling the Fermi energy or the dopant concentration. More importantly, we also note that the Seebeck coefficient extrema of superlattice nanowires have substantially larger magnitudes for Fermi energies near the mini-gaps with only slightly reduced electrical conductivity compared to alloy nanowires (see Fig. 3), which is a direct consequence of the unique potential profile in the transport direction. It is noted that a similar behavior of enhanced Seebeck coefficient due to mini-band formation has also been predicted by Balandin and coworkers for $3 \mathrm{D}$ quantum dot array structures [8]. These observations are significant because they not only illustrate one of the advantages of superlattice nanowires for thermoelectric applications, but they may also account for the enhanced Seebeck coefficient and power factor $\left(S^{2} \sigma\right)$ measured in quantum dot array systems and reported by Harman et al., since both systems have similar periodic potential structures in the direction of carrier transport.

In order to obtain the thermoelectric figure of merit for the superlattice nanowires, the measured bulk heat capacity, sound velocity and Debye temperature of these lead salts are used to determine the thermal conductivity for the nanowire superlattice based on the model calculations mentioned above [9]. Figure 4 shows the calculated $Z T$ as a function of Fermi energy for [001] $\mathrm{PbSe}(5 \mathrm{~nm}) / \mathrm{PbS}(5 \mathrm{~nm})$ superlattice nanowires, and for the corresponding 


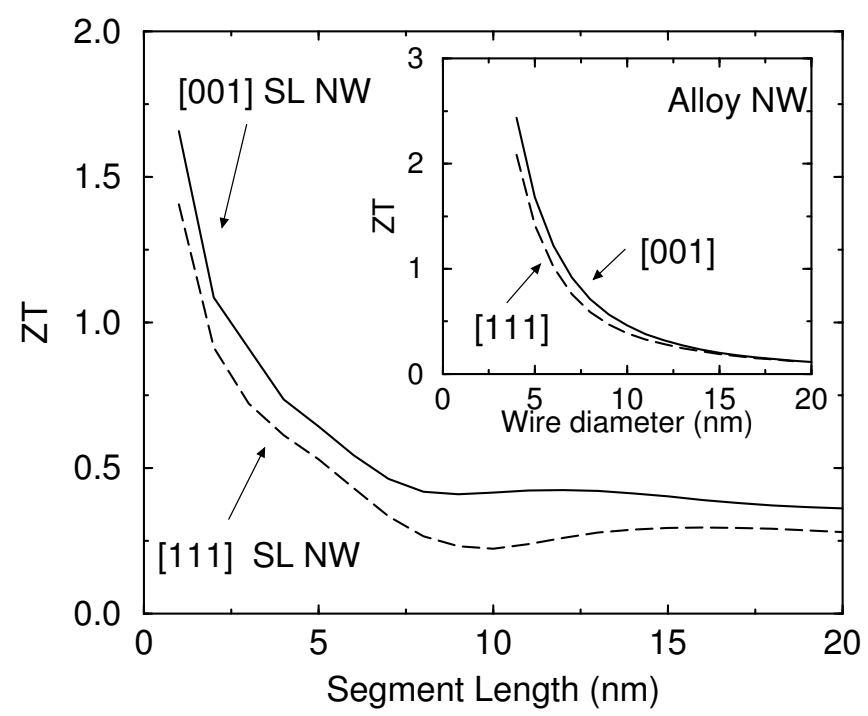

Figure 5: Calculated $Z T$ as a function of segment length for $n$-type 10-nm-diameter $\mathrm{PbSe} / \mathrm{PbS}$ superlattice nanowires of two different crystal orientations at $77 \mathrm{~K}$. Inset: calculated optimal $Z T$ for $\mathrm{PbS}_{0.5} \mathrm{Se}_{0.5}$ alloy nanowires as a function of wire diameter for two different crystal orientations at $77 \mathrm{~K}[10]$.

$\mathrm{PbSe}, \mathrm{PbS}$, and $\mathrm{PbS}_{0.5} \mathrm{Se}_{0.5}$ alloy nanowires with diameters $d_{w}=10 \mathrm{~nm}$ at $77 \mathrm{~K}$, showing an enhancement in $Z T$ for superlattice nanowires over alloy or pure lead salt nanowires. Under an appropriate placement of the Fermi energy, the optimal $Z T$ values in Fig. 4 are 0.65, 0.46, 0.36 , and 0.22 for the $\mathrm{PbSe} / \mathrm{PbS}$ superlattice, $\mathrm{PbS}_{0.5} \mathrm{Se}_{0.5}$ alloy, $\mathrm{PbSe}$, and $\mathrm{PbS}$ nanowires, respectively. As shown in Fig. 4, there are more pronounced $Z T$ extrema as a function of Fermi energy for superlattice nanowires than for homogeneous nanowires, due to the unusual Seebeck coefficient features in superlattice nanowires shown in Fig. 3. It is interesting to note that in Fig. 4 the second $Z T$ maximum for superlattice nanowires corresponds to a positive Seebeck coefficient, whereas the first and the third peaks have negative Seebeck coefficients. Since the sign of the Seebeck coefficient is critical in determining the direction of heat and current flows for thermoelectric devices, this sign should be carefully identified when determining the optimal $Z T$ for the superlattice nanowires.

Figure 5 shows the optimal $Z T$ for $n$-type 10 -nm-diameter $\mathrm{PbSe} / \mathrm{PbS}$ superlattice nanowires as a function of segment length $\left(L_{\mathrm{PbSe}}=L_{\mathrm{PbS}}\right)$ for two crystal orientations ([001] and [111]) at $77 \mathrm{~K}$. For comparison, the inset of Fig. 5 shows the optimal $Z T$ for $\mathrm{PbS}_{0.5} \mathrm{Se}_{0.5}$ alloy nanowires calculated as a function of wire diameter at $77 \mathrm{~K}$, and at a diameter $d_{w}=10 \mathrm{~nm}$, the optimal $Z T$ values are 0.46 and 0.39 for [001] and [111] alloy nanowires, respectively. We note that for both the $\mathrm{PbSe} / \mathrm{PbS}$ superlattice and $\mathrm{PbS}_{0.5} \mathrm{Se}_{0.5}$ alloy nanowires, the nanowires oriented along the [001] direction possess a slightly higher $Z T$ than [111] wires, possibly due to a higher density of states and smaller transport effective masses for [001] wires resulting from the four degenerate pockets. As indicated in Fig. 5, the thermoelectric performance of these 10-nm superlattice nanowires increases rapidly as the segment length decreases, with $Z T$ values higher than those of the corresponding alloy nanowires for segment lengths $\leq 7 \mathrm{~nm}$. However, it is also noted that for longer segment lengths $\left(L_{\mathrm{PbSe}}=L_{\mathrm{PbS}}>7 \mathrm{~nm}\right)$, these superlattice nanowires exhibit a lower $Z T$ than alloy nanowires, which may be due to a lower lattice thermal conductivity in the alloy nanowires. It is interesting to note how 


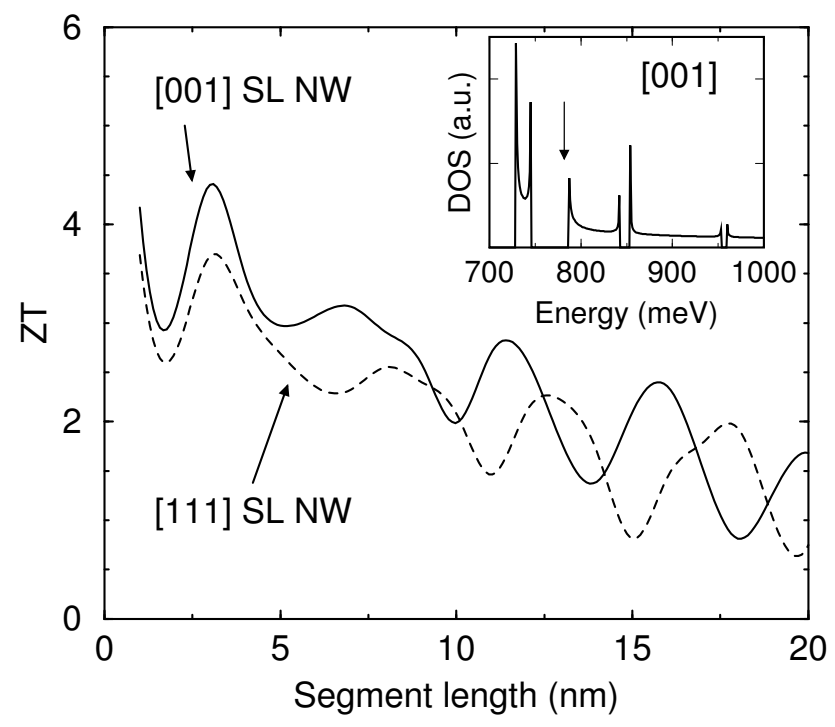

Figure 6: Calculated optimal $Z T$ of $n$-type 5-nm-diameter $\mathrm{PbSe} / \mathrm{PbS}$ superlattice nanowires as a function of segment length $\left(L_{\mathrm{PbSe}}=L_{\mathrm{PbS}}\right)$ for two different crystal orientations at $77 \mathrm{~K}$. Inset: electronic density of states of [001] $\mathrm{PbSe} / \mathrm{PbS}$ superlattice nanowires with a wire diameter of $5 \mathrm{~nm}$ and segment lengths $L_{\mathrm{PbSe}}=L_{\mathrm{PbS}}=10 \mathrm{~nm}$ at $77 \mathrm{~K}$. The arrow indicates the Fermi energy $(\sim 782 \mathrm{meV})$ where the optimal $Z T$ occurs [10].

the thermoelectric performance of these superlattice and alloy nanowires, which contain the same number of $\mathrm{Pb}, \mathrm{S}$, and Se atoms, can be tailored by controlling the spatial arrangement (alloy or periodic segments) of these constituent species.

Figure 6 displays the optimal $Z T$ for $n$-type 5-nm-diameter $\mathrm{PbSe} / \mathrm{PbS}$ superlattice nanowires as a function of segment length $\left(L_{\mathrm{PbSe}}=L_{\mathrm{PbS}}\right)$ for two crystal orientations at $77 \mathrm{~K}$, showing an oscillatory $Z T$ behavior as the segment length varies. For comparison, the optimal $Z T$ for 5-nm-diameter $\mathrm{PbS}_{0.5} \mathrm{Se}_{0.5}$ alloy nanowires is calculated as 1.68 and 1.41 for the [001] and [111] orientations (see inset of Fig. 5), respectively. These $Z T$ oscillations, which are absent or insignificant in larger diameter superlattice nanowires as shown in Fig. 5, are due to the larger subband separation as the wire diameter decreases. For example, the inset of Fig. 6 depicts the density of states for a 5-nm-diameter $\mathrm{PbSe}(10 \mathrm{~nm}) / \mathrm{PbS}(10 \mathrm{~nm})$ superlattice nanowire oriented along the [001] direction, showing that only one subband contributes to the transport properties in the energy range of importance, since the onset of the second subband does not appear until higher energies. In contrast, the density of states of 10-nm-diameter PbSe/PbS superlattice nanowires exhibits a much more complicated energy dependence due to the superposition of multiple subbands in the energy range of optimal $Z T$. Therefore, for small diameter superlattice nanowires, their transport properties are mainly determined by the band width of the mini-bands and the mini-gaps that are highly dependent on the segment length, instead of the diameter-dependent subband energies. As shown in Fig. 2, the mini-band and the mini-gap structure of one subband evolves from that of the alloy limit to the classical limit as the segment length increases, resulting in an oscillatory optimal $Z T$ due to the variation in the widths and gaps of the mini-bands. However, for larger-diameter superlattice nanowires, this $Z T$ oscillation as a function of segment length becomes smeared out due to the averaging effect of the contributions from multiple subbands. In Fig. 6, the optimal segment length for 5-nm-diameter $\mathrm{PbSe} / \mathrm{PbS}$ 
Table 2: Optimal segment lengths and the highest $Z T$ of 5 -nm-diameter $n$ and $p$-type lead salt superlattice nanowires $(\mathrm{PbSe} / \mathrm{PbS}$ and $\mathrm{PbSe} / \mathrm{PbTe}$ ) at $77 \mathrm{~K}$.

\begin{tabular}{c|c|cc|cc}
\hline \hline \multicolumn{2}{c|}{ material } & \multicolumn{2}{|c|}{ PbSe/PbS SLNW } & \multicolumn{2}{|c}{ PbSe/PbTe SLNW } \\
\hline \multicolumn{2}{c|}{ orientation } & {$[001]$} & {$[111]$} & {$[001]$} & $[11]]$ \\
\hline$n$-type & optimal $Z T$ & 4.4 & 3.7 & 6.37 & 8.07 \\
& Segment Length & $3 \mathrm{~nm}$ & $3 \mathrm{~nm}$ & $2 \mathrm{~nm}$ & $2 \mathrm{~nm}$ \\
\hline$p$ type & optimal $Z T$ & 6.15 & 4.45 & 6.02 & 7.45 \\
& Segment Length & $2 \mathrm{~nm}$ & $2 \mathrm{~nm}$ & $2 \mathrm{~nm}$ & $2 \mathrm{~nm}$ \\
\hline \hline
\end{tabular}

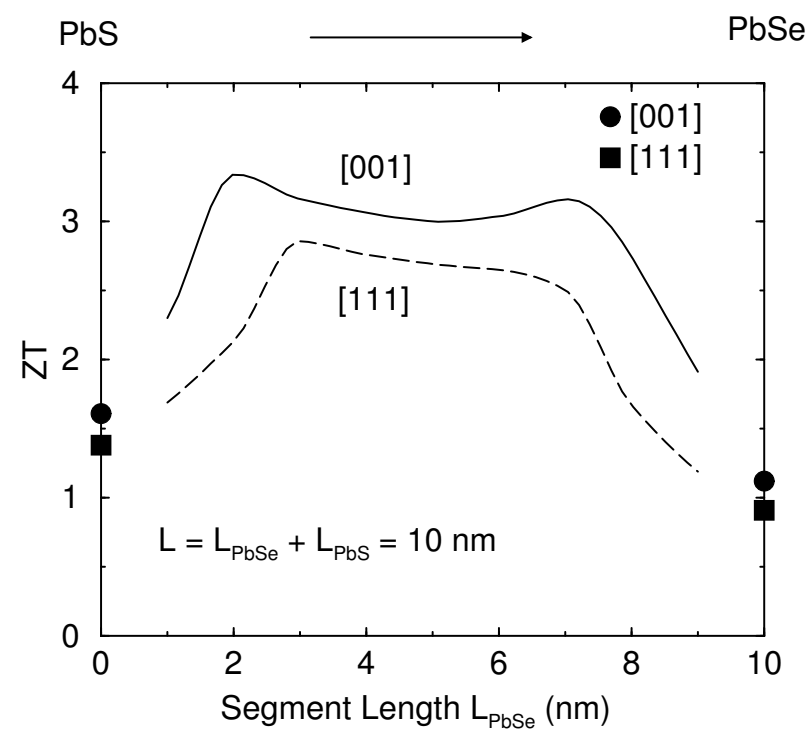

Figure 7: Calculated optimal $Z T$ for 5-nm-diameter $n$-type $\mathrm{PbSe} / \mathrm{PbS}$ superlattice nanowires as a function of $\mathrm{PbSe}$ segment length $L_{\mathrm{PbSe}}$ at $77 \mathrm{~K}$ with different nanowire crystalline orientations. The segment length of $\mathrm{PbS}$ is chosen such that the period $L=L_{\mathrm{PbSe}}+L_{\mathrm{PbS}}=10 \mathrm{~nm}$ is constant. The optimal $Z T$ for 5-nm-diameter $\mathrm{PbS}$ and $\mathrm{PbSe}$ 1D nanowires are shown as circles and squares for [001] and [111] orientations, respectively $[10]$.

superlattice nanowires is about $3 \mathrm{~nm}$ for both [001] and [111] orientations, with optimal $Z T$ values of 4.4 and 3.7, respectively, which are much higher than the optimal $Z T$ of 10-nm-diameter superlattice nanowires.

Since the lead salts have valence band structures similar to their conduction bands, $p$-type lead salts superlattice nanowires are expected to exhibit thermoelectric performance similar to their $n$-type counterparts. The results for the $p$-type $\mathrm{PbSe} / \mathrm{PbS}$ superlattice nanowires have optimal $Z T$ values slightly higher than for the $n$-type wires, and this may be due to the smaller effective masses for holes than for electrons in the lead salts. Table 2 summarized the optimal $Z T$ ( $n$ and $p$ types) and the corresponding segment lengths for $5 \mathrm{~nm}$ diameter $\mathrm{PbSe} / \mathrm{PbS}$ and $\mathrm{PbSe} / \mathrm{PbTe}$ superlattice nanowires at $77 \mathrm{~K}$ [10]. Here it is seen that $\mathrm{PbSe} / \mathrm{PbTe}$ superlattice nanowires generally possess a higher $Z T$ than for $\mathrm{PbSe} / \mathrm{PbS}$, which results from the smaller effective masses and higher mobilities for carriers in $\mathrm{PbTe}$ relative to $\mathrm{PbS}$. 
The calculations shown so far are made assuming an equal segment length for the two constituent materials. However, for a given period length $L=L_{A}+L_{B}$ for superlattice nanowires, the thermoelectric performance may be further optimized by adjusting the length ratio of the two materials. As an example, Fig. 7 shows the calculated $Z T$ for 5 -nm-diameter $n$-type $\mathrm{PbSe} / \mathrm{PbS}$ superlattice nanowires as a function of $\mathrm{PbSe}$ segment length $L_{\mathrm{PbSe}}$ at a given period $L=L_{\mathrm{PbSe}}+L_{\mathrm{PbS}}=10 \mathrm{~nm}$ along two crystal orientations at $77 \mathrm{~K}$. The optimal $Z T$ of 5-nm-diameter PbSe and $\mathrm{PbS}$ nanowires at $77 \mathrm{~K}$ are also shown in Fig. 7 for [001] and [111] orientations as circles and squares, respectively. From Fig. 7, we see that a higher $Z T$ can be achieved for $\mathrm{PbSe} / \mathrm{PbS}$ superlattice nanowires with unequal segment lengths for $\mathrm{PbSe}$ and $\mathrm{PbS}$ nanodots. For [001] orientations, $\mathrm{PbSe}(2 \mathrm{~nm}) / \mathrm{PbS}(8 \mathrm{~nm})$ superlattice nanowires have the highest $Z T$ of 3.31 , while $\mathrm{PbSe}(3 \mathrm{~nm}) / \mathrm{PbS}(7 \mathrm{~nm})$ superlattice nanowires are optimal for the [111] orientation with $Z T \sim 1.8$. As a comparison, for $L_{\mathrm{PbSe}}=L_{\mathrm{PbS}}=$ $5 \mathrm{~nm}$, the optimal $Z T$ values of corresponding [001] and [111] superlattice nanowires are calculated as 2.98 and 1.68 , respectively.

Since the lattice constants of these lead salts are about $5 \AA$, there are $\sim 400$ unit cells in a nanodot with a diameter of $5 \mathrm{~nm}$ and segment length of $2 \mathrm{~nm}$, validating the application of the effective mass theorem and the band structure parameters used in the model calculation. These superlattice nanowires are not expected to be in the alloy limit, and they do possess a unique electronic band structure that is dramatically different from that for a simple nanowire. This unique band structure partly accounts for their superior performance when compared to their corresponding alloy nanowires. Recently, Bjork and coworkers have fabricated $\mathrm{InP} / \mathrm{InAs}$ superlattice nanowires with atomically perfect interfaces by chemical beam epitaxy [13] for segment lengths as short as $1.5 \mathrm{~nm}$, indicating that the proposed superlattice nanowire structures that are here discussed for thermoelectric applications may be achieved experimentally. Experimental studies of these nanowires are needed to check the validity of the assumptions made in model calculations such as are given here, and to further improve the values of the parameters used in these model predictions.

\section{Concluding Remarks}

Over the past decade, it has been shown by various groups that low dimensional thermoelectrics offer promise for the possibility of fabricating thermoelectric materials with enhanced $Z T$ values. Model calculations and specially designed experiments bring confidence to the reliability of the model calculations [16]. While localization effects become important for $4 \mathrm{~nm} \mathrm{Bi} \mathrm{nanowires} \mathrm{they} \mathrm{are} \mathrm{not} \mathrm{important} \mathrm{for} 9 \mathrm{~nm}$ diameter, allowing for sufficient flexibility in variation of wire diameter and upon alloying with Sb to achieve ranges of $Z T$ that should be of interest for practical applications [16].

However, the model systems that have been considered in terms of establishing proofof-principle and technical feasibility are generally not practical for commercial applications insofar as these systems are too expensive or complex for mass production, too small in total cooling power or in energy generation, or unsuitable for scale-up. Therefore new research in low dimensional thermoelectrics should be directed toward approach which can be selfassembled into macroscopic systems while having low dimensional nanosized constituents which show strong interface phonon scattering but preservation of the electronic power factor. Examples of structures we have in mind are nanoparticles or nanowires of material A embedded in a host material B [17]. Advances in the synthesis of self-assembled templates, 
such as anodic alumina templates, but with lower thermal conductivity are needed as well as the development of filling methods based on electrochemistry which are highly flexible, have high filling factor efficiency and yield highly crystalline materials with high mobilities. Significant progress in this direction has recently been reported [18].

Some by-product of low dimensional thermoelectric materials research has been the development of materials and technologies exhibiting new physical phenomena not previously observed. Two examples of such advances is the synthesis of anti-dot films of bismuth based on an anodic alumina template substrate which exhibits a large enhancement in the $2 \mathrm{D}$ weak antilocalization effect [19]. A second example is the observation of a sharp and strong feature in the optical absorption of bismuth nanowires, but not observed in bulk bismuth, and attributed to an indirect transition between $L$-point and $T$-point states in bismuth nanowire [20]. We can expect thus that research in low dimensional thermoelectric materials is likely to yield desirable outcomes from both a practical and scientific standpoint.

The authors wish to thank Professor Gang Chen for valuable discussions and NASA for support of this research.

\section{References}

[1] L. D. Hicks and M. S. Dresselhaus, Phys. Rev. B 47, 12727-12731 (1993).

[2] L. D. Hicks and M. S. Dresselhaus, Phys. Rev. B 47, 16631-16634 (1993).

[3] R. Venkatasubramanian, E. Siivola, T. Colpitts, and B. O’Quinn, Nature 413, 597 (2001).

[4] Y.-M. Lin, X. Sun, and M. S. Dresselhaus, Phys. Rev. B 62, 4610-4623 (2000).

[5] M. S. Dresselhaus, Yu-Ming Lin, S. B. Cronin, O. Rabin, M. R. Black, G. Dresselhaus, and T. Koga. In Semiconductors and Semimetals: Recent Trends in Thermoelectric Materials Research III, edited by T. M. Tritt, pages 1-121, Academic Press, San Diego, CA, 2001. Chapter 1.

[6] T. C. Harman, P. J. Taylor, M. P. Walsh, and B. E. LaForge, Science 297, 2229-2232 (2002).

[7] L. Piraux, I. M. George, J. F. Despres, C. Leroy, E. Ferain, R. Legras, K. Ounadjela, and A. Fert, Appl. Phys. Lett. 65, 2484-2486 (1994).

[8] A. A. Balandin and O. L. Lazarenkova, Appl. Phys. Lett. 82, 415 (2003).

[9] C. Dames and G. Chen, (2003). J. Appl. Phys.(to be published).

[10] Yu-Ming Lin and M. S. Dresselhaus, Phys. Rev. B 68, 075304(1-14) (2003).

[11] Y. Wu, R. Fan, and P. Yang, Nano Lett. 2, 83 (2002).

[12] M. S. Gudiksen, L. J. Lauhon, J. Wang, D. C. Smith, and C. M. Lieber, Nature 415, 617-620 (2002).

[13] M. T. Bjork, B. J. Ohlsson, T. Sass, A. I. Persson, C. Thelander, M. H. Magnusson, K. Deppert, L. R. Wallenberg, and L. Samuelson, Nano Lett. 2, 87-89 (2002).

[14] R. Dalven, Infrared Physics 9, 141-184 (1969).

[15] R. S. Allgaier and W. W. Scanlon, Phys. Rev. 111, 1029 (1958).

[16] J. P. Heremans, MRS Fall meeting, 2003 paper S1.1.

[17] R. Yang and G. Chen, MRS Fall meeting, 2003 paper S5.2.

[18] O. Rabin, G. Chen and M.S. Dresselhaus, MRS Fall meeting, 2003 paper S5.4.

[19] O. Rabin, M.S. Dresselhaus, MRS Fall meeting, 2003 paper L12.1.

[20] M. R. Black, P. L. Hagelstein, S. B. Cronin, Y.-M. Lin, and M. S. Dresselhaus, Phys. Rev. B 68 (2003). 